\title{
Ein Aufstand und ein Bärendienst am Gesundheitswesen
}

Liebe Leserinnen und Leser,

der 17. Juni war nicht nur in der Vergangenheit ein markantes Datum, sondern scheint es auch in der Gegenwart zu sein. An diesem Tag im Jahr 2010 wurde durch den Gemeinsamen Bundesausschuss (G-BA) beschlossen, dass Glinide und Glitazone von der Verordnungsfähigkeit zulasten der gesetzlichen Krankenversicherungen ausgeschlossen werden. Bei den Gliniden wird als Grund des Ausschlusses der nicht belegte Nutzen genannt, jedoch kann der Wirkstoff Repaglinid bei schwerer Niereninsuffizienz weiter verordnet werden. Dieser Vorgang ist von anderen Präparaten in der Vergangenheit bekannt. Bei den Glitazonen argumentiert der G-BA, dass bei der Nutzenbewertung das deutlich belegte Schadenspotenzial der Grund für den Verordnungsausschluss sei. Für diese Stoffgruppe gibt es auch keine Ausnahmeregelung. In den nächsten Monaten hätten alle Patienten auf Alternativpräparate umgestellt werden müssen. Allerdings hat das Bundesministerium für Gesundheit den Verordnungsausschluss der Glitazone nun erst einmal blockiert und fordert eine ergänzende Stellungnahme des G-BA (s. S. 57, Anm. d. Red.).

\section{Der G-BA im argumentativen Alleingang?}

Als Arzt, der die Glitazone nur sehr selten und nur in sehr definierten Indikationen verordnet hat, bedeutet dies keine wesentliche Beeinträchtigung der Tätigkeit. Jedoch sollte dieser sehr weit reichende Beschluss an diesem historischen Tag der deutschen Geschichte unter verschiedenen Gesichtspunkten noch einmal betrachtet werden. Der G-BA hat mit Schreiben vom 22.2.2005 das Institut für Qualität und Wirtschaftlichkeit im Gesundheitswesen (IQWiG) beauftragt, eine Nutzenbewertung von Glitazonen zu erstellen, die dann im November 2008 publiziert wurde. Darin kommt das IQWiG zu dem Schluss:,„Für die Gesamtrate unerwünschter Ereignisse zeigte sich kein Nutzen, Zusatznutzen oder
Schaden einer Behandlung mit einem Glitazon." Wie kann es sein, dass der G-BA so stark von der Bewertung des IQWiG abweicht?

Ein weiterer Punkt ist bedenkenswert. Welche Rolle spielt denn das Bundesinstitut für Arzneimittel und Medizinprodukte (BfArM)? Wenn die Gefahren durch Glitazone so groß sind, dass die gesetzlich versicherten Patienten durch den G-BA geschützt werden müssen, warum setzt das BfArM künftig privat versicherte Patienten diesen Gefahren auch weiterhin aus?

\section{An Alternativen bleibt nicht viel}

Weiterhin spricht der G-BA in seiner Verlautbarung davon, dass andere Präparate zur Verfügung stehen, die derartige unerwünschte Nebenwirkungen und Langzeitschäden nicht haben. Doch welche Präparate sind das? Meist werden Personen mit Typ-2-Diabetes zuerst Metformin und Sulfonylharnstoffe in Mono- bzw. Kombinationstherapie verordnet. Wenn nun die Glinide nur noch in Ausnahmefällen verordnungsfähig sind, was steht dann zur Verfügung? Die neuen Wirkprinzipien der DPP-4-Inhibitoren sind dann die einzigen Alternativen, und auch im Preis sind diese den Glitazonen vergleichbar. Doch gilt das nur kurzfristig, denn die Glitazone werden in Kürze aus dem Patentschutz laufen und als Generika deutlich günstiger auf dem Markt verfügbar sein. Dies wäre ein kleiner Schritt zur Senkung der Medikamentenkosten im Gesundheitswesen gewesen.

Der 17. Juni ist somit nicht nur der Tag des Aufstandes der Arbeiter in der DDR, sondern auch der Tag des Aufstands des G-BA gegen das IQWiG, das BfArM und die Kostensenkung im Gesundheitswesen.

Stephan Martin

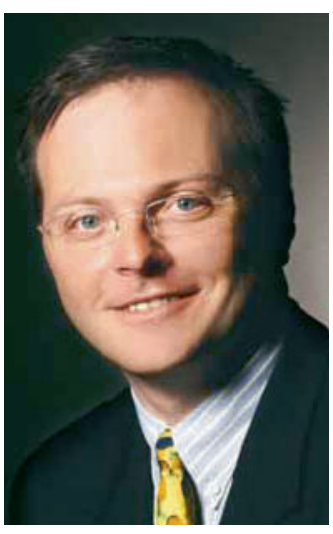

Prof. Dr. med. Stephan Martin, Ärztlicher Direktor des Westdeutschen Diabetes- und Gesundheitszentrums an den Sana Kliniken Düsseldorf und Herausgeber von Info Diabetologie 